\title{
Dual-band optical coherence tomography using a single supercontinuum laser source
}

Siyu Chen

Xiao Shu

Ji Yi

Amani Fawzi

Hao F. Zhang 


\title{
Dual-band optical coherence tomography using a single supercontinuum laser source
}

\author{
Siyu Chen, ${ }^{\mathrm{a}}$ Xiao Shu, ${ }^{\mathrm{a}}$ Ji Yi, ${ }^{\mathrm{a}, \mathrm{b}}$ Amani Fawzi, ${ }^{\mathrm{c}}$ and Hao F. Zhang ${ }^{\mathrm{a}, \mathrm{c}, *}$ \\ ${ }^{a}$ Northwestern University, Department of Biomedical Engineering, 2145 Sheridan Road, Evanston, Illinois 60208, United States \\ ${ }^{\mathrm{b} B}$ Boston University, Department of Medicine, 650 Albany Street, Boston, Massachusetts 02118, United States \\ 'Northwestern University, Department of Ophthalmology, 300 East Superior Street, Chicago, Illinois 60611, United States
}

\begin{abstract}
We developed a simultaneous visible-light (Vis) and near-infrared (NIR) dual-band optical coherence tomography (OCT) system using a single supercontinuum laser source. The goal was to benchmark our newly developed Vis-OCT against the well-developed NIR-OCT. The Vis-OCT subsystem operated at $91 \mathrm{~nm}$ full-widthat-half-maximum (FWHM) bandwidth centered at $566 \mathrm{~nm}$; the NIR-OCT subsystem operated at $93 \mathrm{~nm}$ FWHM bandwidth centered at $841 \mathrm{~nm}$. The axial resolutions were 1.8 and $4.4 \mu \mathrm{m}$ in air for the Vis- and NIR-OCT subsystems, respectively. We compared the respective performances, including anatomical imaging, angiography, absolute retinal blood flow measurements, and spectroscopic analysis for retinal blood oxygen saturation $\left(\mathrm{sO}_{2}\right)$, between the two subsystems in rodents in vivo. While demonstrating minor discrepancies related to operation wavelengths, both subsystems showed comparable performances in the first three tests. However, we were only able to retrieve $\mathrm{SO}_{2}$ using the Vis-OCT subsystem. $\odot 2016$ Society of Photo-Optical Instrumentation Engineers (SPIE) [DOI: 10.1117/1 .JBO.21.6.066013]
\end{abstract}

Keywords: optical coherence tomography; dual-band; optical coherence tomography oximetry; optical coherence tomography flowmetry.

Paper 160089RR received Feb. 15, 2016; accepted for publication May 20, 2016; published online Jun. 15, 2016.

\section{Introduction}

Optical coherence tomography (OCT) is a low-coherence interferometry-based technique. It can provide three-dimensional tomographic information with micrometer spatial resolution and millimeter imaging depth, fulfilling significant applications in both scientific researches and clinical practices. Its major strength, however, lies in ophthalmic imaging. ${ }^{1}$ The anatomical structure of the eye permits unhindered access of the probing light into the retina, rendering OCT fundus images with explicit details. More importantly, OCT can be further functionalized. For example, OCT angiography can enhance the contrast of microvessels for morphological-based diagnosis, while Doppler OCT measures blood flow for hemodynamics-related applications.

Until recently, most ophthalmic OCT systems operate within the near-infrared (NIR) range of the electromagnetic spectrum. Several factors have supported this choice. First, biological tissues (blood, cell matrix, melanin, and so on) exhibit minimal optical absorption and scattering within NIR range among usable optical bands. ${ }^{2}$ Therefore, the illumination beam is both less attenuated and less diffused, resulting in greater penetration depth and less blurred images. Second, the accessibility and economy of NIR broadband light sources was another important driving factor for the adoption of NIR-OCT. ${ }^{3}$

Unfortunately, NIR-OCT alone may not provide all the information that researchers and physicians are interested in. Among them, blood oxygen saturation $\left(\mathrm{sO}_{2}\right)$ within the retinal circulation is one important physiological indicator. ${ }^{4}$ Theoretically, OCT can noninvasively measure blood $\mathrm{sO}_{2}$ based on the distinctive absorption spectra contrast between oxygenated $\left(\mathrm{HbO}_{2}\right)$ and

*Address all correspondence to: Hao F. Zhang, E-mail: hfzhang@ northwestern edu deoxygenated (HbR) hemoglobin. ${ }^{5,6}$ However, optical absorption is considerably reduced in the NIR region and is often overshadowed by tissue scattering. As a result, NIR-OCT may not be ideal if accurate quantification of $\mathrm{sO}_{2}$ is desired, which was first reported by Faber and van Leeuwen and later verified numerically by Chen et al. ${ }^{7,8}$

Visible-light OCT (Vis-OCT) provides a solution. Within the spectral range from 500 to $600 \mathrm{~nm}, \mathrm{HbO}_{2}$ and $\mathrm{HbR}$ absorption spectra share multiple isosbestic points. ${ }^{9}$ More importantly, blood optical absorption contrast increases by over 10-fold as compared to that in the NIR region. These advantages collectively make Vis-OCT oximetry possible, as have been demonstrated in ex vivo, in situ, and in vivo experiments. ${ }^{10-12}$ It was also reported that Vis-OCT $\mathrm{sO}_{2}$ measurements from rodent retina circulation can achieve high accuracy within $\pm 4 \%$ relative error, ${ }^{13}$ while $\mathrm{sO}_{2}$ change due to oxygen unloading can be observed. ${ }^{14,15}$ In addition, utilizing a shorter wavelength range, Vis-OCT intrinsically has higher axial resolution than NIR-OCT with comparable bandwidth, which can further facilitate its oximetry application. ${ }^{16}$

Despite Vis-OCT's successful demonstrations, there are still concerns left unaddressed. One major question is whether the elevated tissue absorption and scattering in the visible spectrum range will adversely affect its imaging capability, both anatomically and functionally. Thus, it is worthwhile to compare the two imaging modalities in a single combined system side-by-side. In addition, Vis- and NIR-OCT may possess distinctive imaging contrasts due to different tissue optical response. An uninformed physician may find the discrepancy confusing; thus, it must be investigated before Vis-OCT can be used clinically. 
In this work, we seek to answer these questions using a visible-light and NIR dual-band OCT. We simultaneously acquired both Vis- and NIR-OCT images over the same region of interest. Discrepancies between Vis- and NIR-OCT images were identified, whose major indicators included anatomical features and imaging resolution. We further compared their functional imaging abilities to measure retinal blood $\mathrm{sO}_{2}$ and absolute blood flow.

\section{Materials and Methods}

\subsection{System Setup}

Figure 1 shows the schematic diagram of our dual-band OCT system. A supercontinuum laser (SuperK EXTREME, NKT photonics) served as the sole light source. A pair of dichroic mirrors (DM1 and DM2, SuperK SPLIT, NKT photonics) selectively separated the output light into visible (500 to $650 \mathrm{~nm}$ ) and NIR (780 to $950 \mathrm{~nm}$ ) bands. We then individually delivered the separated visible and NIR beams into the input arms of two Michelson interferometers. Each interferometer was independently optimized. The Vis-OCT interferometer adopted a freespace configuration. The 50:50 beam splitter (Thorlabs) delivered equal amount of light energy toward the sampling arm and the reference arm. In NIR-OCT, we used conventional $2 \times 2$ optical fiber coupler (Thorlabs) with even split ratio. Existing fiber couplers do not perform well within the visible-light spectral range. Although optical dispersion caused by imperfect fiber couplers can potentially be corrected numerically, such correction can also introduce additional artifacts. Therefore, we used the free-space design here for optimal performance in the VisOCT subsystem.

The sampling beams of the Vis- and NIR-OCT subsystems were recombined by a third dichroic mirror (DM3, Semrock) and shared identical optical delivery path for retinal imaging. To project scanning patterns onto mouse retina, we used a pair of galvanometer mirrors (Nutfiled Technology) and a 5:1 Keplerian telescope. The collimated output beam has a Gaussian beam diameter of $0.2 \mathrm{~mm}$ for both Vis- and NIR-OCTs. In order to compensate the chromatic aberration across two wavelength bands, we adjusted the collimating lens (CL) in the NIR-OCT subsystem to ensure both probing beams focused onto the same focal plane within the retina. The two subsystems had

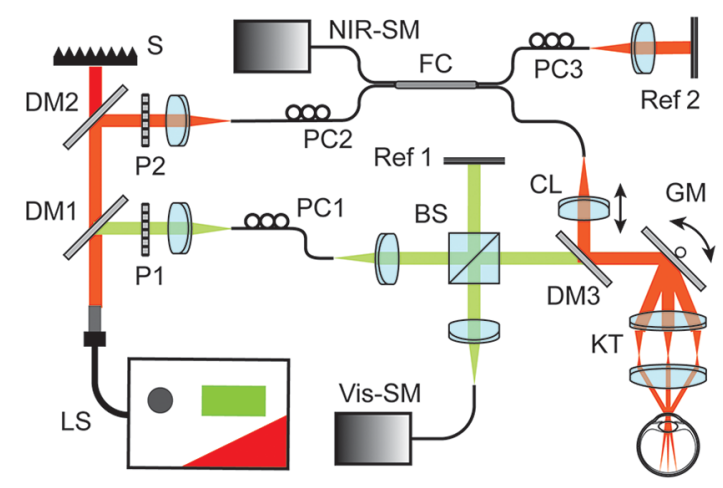

Fig. 1 Dual-band OCT system schematic diagram. BS, beam splitter; $\mathrm{CL}$, collimating lens; DM1 to DM3, dichroic mirrors; FC, $2 \times 2$ fiber coupler; GM, galvanometer scan mirrors; KT, Keplerian telescope; LS, supercontinuum laser source; $\mathrm{P}$, polarizer; $\mathrm{PC}$, polarization controller; Ref 1 and Ref 2, reference arms; S, beam stopper; SM, spectrometer. independent reference arms. Within each reference arm, we carefully balanced dispersion mismatch and attenuated the reflected beam intensity for optimal imaging quality.

Two home-made spectrometers digitized the interference spectra. The Vis-OCT spectrometer was calibrated to cover the spectral range from 513 to $620 \mathrm{~nm}$. In the NIR-OCT spectrometer, the calibrated wavelength range was from 773 to $909 \mathrm{~nm}$. The spectral resolution is $0.052 \mathrm{~nm}$ for the visible spectrometer and $0.066 \mathrm{~nm}$ for the NIR spectrometer. As the spectrum intensity fell at both edges, full-width-at-half-maximum (FWHM) bandwidth was 91 and $93 \mathrm{~nm}$ for Vis- and NIR-OCT bands, respectively.

\subsection{Performance Characterization}

We placed a silver mirror in the sampling arm where the mouse eye would otherwise be located. It created a single reflective surface, which corresponded to an axial impulse response. One additional neutral density (ND) filter (Thorlabs, optical density $=3.0$ ) was inserted to attenuate the light so that the detected signal fell within the dynamic range of the spectrometer. We adjusted the location of the reference mirrors to change the relative path length difference between the sampling and reference beams. The manipulation was analogous to imaging the sample at various depths. At each location, we recorded the interference fringes from the spectrometers for further analysis.

\subsection{Animal Preparation}

We imaged the retina of wild-type C57BL/6 mice to compare the imaging capability (actual image resolution, anatomic features, and functional blood flow and $\mathrm{sO}_{2}$ measurements) between Vis- and NIR-OCT in vivo. The mouse was anesthetized using Ketamine/Xylazine cocktail solution (11.45 mg Ketamine and $1.71 \mathrm{mg}$ Xylazine per $\mathrm{ml}$ of solution, respectively) injected intraperitoneally. The dosage was $87 \mathrm{mg}$ Ketamine and $13 \mathrm{mg}$ Xylazine per kilogram of body weight.

Once the mouse entered the desired anesthesia plane, we applied $1 \%$ Tropicamide ophthalmic solution to dilate its iris. It was subsequently transferred to a home-made imaging stage and properly immobilized for imaging. During the process, we applied artificial tears every few minutes and in the intervals of acquisitions to prevent eye dehydration. After the experiment, the mouse was allowed to recover and then sent back to the animal housing facility. All procedures were approved by the Northwestern University IACUC and conformed to the Association for Research in Vision and Ophthalmology Statement on Animal Research.

\subsection{Imaging Protocols}

We simultaneously acquired Vis- and NIR-OCT images in a single volumetric scan. Depending on the applications, the imaging procedure used one of the following two scanning protocols. To obtain OCT anatomical imaging, angiography, and retinal blood $\mathrm{sO}_{2}$ analysis, we used the raster scanning protocol. It covered a square field of view of $0.8 \mathrm{~mm}$ by $0.8 \mathrm{~mm}$ on the mouse retina. During each acquisition, we took a group of five repetitive unidirectional B-scans at the same location before moving to the next. Each B-scan consisted of 400 A-lines acquired at $50 \mathrm{kHz}$. A total of $512 \mathrm{~B}$-scan locations were sequentially acquired. The entire volumetric scan took $\sim 25.4$ s to complete. 
The other scanning protocol facilitated calculation of absolute blood flow within the retina circulation. We adopted the dual-ring scanning trajectory centered at optic nerve head to rapidly obtain both Doppler phase shift $(\Delta \varphi)$ and Doppler angle $\left(\Theta_{d}\right) .{ }^{17}$ The diameters of the inner and outer rings were 0.52 and $0.63 \mathrm{~mm}$, respectively. Each ring consisted of $4096 \mathrm{~A}-$ lines acquired at $70 \mathrm{kHz}$. The two ring-scan trajectories were repeated eight times alternatingly, which served to average the pulsatile flow pattern. The acquisition time was $0.9 \mathrm{~s}$.

\subsection{Data Processing}

Our imaging protocol allowed us to extract anatomical OCT images, angiography, and retinal vessel blood $\mathrm{sO}_{2}$ from the same volumetric data set using the raster scanning protocol. We recovered the complex OCT signal using full-spectrum Fourier transform. After bulk motion correction, ${ }^{18}$ OCT anatomical images were generated by averaging amplitude B-scans within the same cross-sectional location. Meanwhile, calculating intragroup decorrelation on complex OCT B-scans yielded OCT angiography. ${ }^{19}$

The detailed steps to perform spectroscopic OCT analysis for $\mathrm{sO}_{2}$ were previously documented. ${ }^{12}$ Briefly, we adopted shorttime Fourier transform (STFT), which split the entire spectrum into equally spaced narrow bands in k-space. The Gaussian window size was $0.23 \mu \mathrm{m}^{-1}$ for the visible band and $0.14 \mu \mathrm{m}^{-1}$ for the NIR band. This gave us STFT bandwidths of $12 \mathrm{~nm}$ at $566 \mathrm{~nm}$ for Vis-OCT and $16 \mathrm{~nm}$ at $841 \mathrm{~nm}$ for NIR-OCT, relaxing the axial resolution for visible and NIR images to 11.7 and $19.4 \mu \mathrm{m}$, respectively. Each narrow-band spectrum was then individually reconstructed to OCT amplitude images, where image segmentation was performed to extract OCT intensity from the bottom vessel wall. We used the same modified Beer-Lambert's law to model the detected wavelength-dependent OCT intensity as in Ref. 12. After mathematical treatment, a linear relationship between the measured OCT amplitude and relative $\mathrm{HbO}_{2} / \mathrm{HbR}$ concentration can be established. ${ }^{12}$ Thus, we recovered blood $\mathrm{sO}_{2}$ by least-squares fitting the obtained STFT OCT intensity spectrum from the target vessel.

OCT flow analysis was performed on the dual-ring scanning data set. After necessary preprocessing, complex OCT signal was reconstructed along the scanning trajectory. We then estimated $\Theta_{d}$ and $\Delta \varphi$ for each identified vessel using the corresponding OCT structural and phase image, respectively. ${ }^{17}$ When phase-wrapping was observed, we applied correction algorithm to resolve any $2 \pi$ ambiguity before calculating flows. ${ }^{20}$

\section{Results}

\subsection{System Sensitivity and Imaging Resolution}

The intensities of sampling beams were measured using a calibrated integrating sphere (Ocean Optics), which were 0.9 and $0.8 \mathrm{~mW}$ for the visible and NIR bands, respectively. Figure 2 plots the mirror-surface impulse response versus imaging depth for the two subsystems. We used the first peaks as the references for sensitivity and resolution estimation. The relative height with respect to the noise floor was $26.6 \mathrm{~dB}$ for Vis-OCT and $38.2 \mathrm{~dB}$ for NIR-OCT. Taking the 60 -dB round-trip attenuation from the ND filter into consideration, we calculated the sensitivity for the Vis- and NIR-OCT to be 86.6 and $98.2 \mathrm{~dB}$, respectively. FWHM $(-6 \mathrm{~dB})$ for the first two peaks served as the benchmarks for axial resolutions. The Vis-OCT subsystem showed an axial
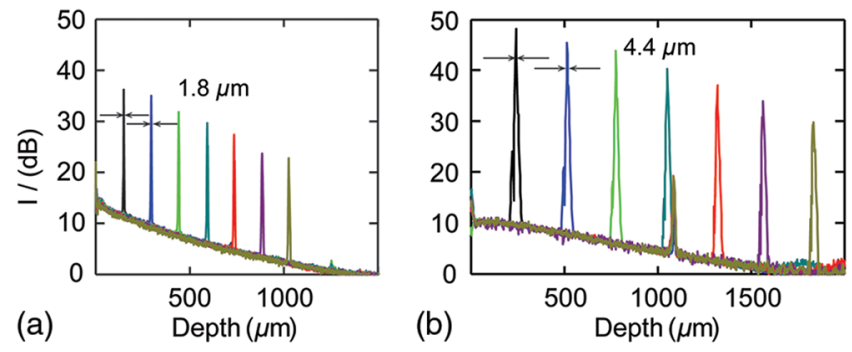

Fig. 2 System sensitivity and resolution characterization. (a) Axial impulse responses at different depths for Vis-OCT subsystem. FWHM widths for the first two peaks were $1.8 \mu \mathrm{m}$. (b) Axial impulse responses at different depths for NIR-OCT subsystem. FWHM widths for the first two peaks were $4.4 \mu \mathrm{m}$.

resolution of $1.8 \mu \mathrm{m}$ in air. Its resolution was more than twofold higher than our NIR-OCT with comparable bandwidth, which was $4.4 \mu \mathrm{m}$. We also characterized sensitivity roll-off by linear fitting all the peaks in the series, which was $16.4 \mathrm{~dB} / \mathrm{mm}$ for Vis-OCT and $12.2 \mathrm{~dB} / \mathrm{mm}$ for NIR-OCT.

When imaging mouse fundus, we estimated the effective numerical aperture to be $\sim 0.05$ for both Vis and NIR subsystems. It would give us the theoretical lateral resolutions of 7.1 and $10.5 \mu \mathrm{m}$ at their respective center wavelengths. However, due to the optical quality of rodent eye, actual resolution during in vivo imaging will not achieve the theoretical values. ${ }^{21}$

\subsection{Comparison of Imaged Anatomic Features Between Vis- and NIR-OCT}

Figure 3 compares the OCT anatomical and angiographic images obtained by Vis- and NIR-OCT subsystems. We projected mean pixel intensity along depth axis across the entire retina thickness to generate en face OCT fundus images [Figs. 3(a) and 3(b)]. Vis- and NIR-OCT images demonstrate similar features, where retinal blood vessels, neural fiber layer (NFL), and retinal pigment epithelium (RPE) contribute to the majority of the contrast. In addition, both OCT angiograms [Figs. 3(c) and 3(d)] show comparable vascular structures and topographies. We overlaid the anatomical and angiographic Bscans in Figs. 3(e) and 3(f), which were taken from the location indicated by the yellow dashed line in Figs. 3(a) and 3(b), respectively. Multiple retinal layers are distinguishable in both images, with NFL and RPE the brightest. Furthermore, the OCT angiographs correspond well with their corresponding structure B-scans, showing distribution of vessels mainly around NFL, inner border of the inner nuclear layer (INL), inner border of the outer plexiform layer (OPL), and choroid.

Apart from similarities, Vis- and NIR-OCT also demonstrated considerable difference in image contrast. We can see that a strip pattern, which originates from the axons of retinal ganglion cells in NFL, is more recognizable in the Vis-OCT fundus image. The major contributing factor is the difference in penetration depth between the two wavelength ranges. As NIR sampling beam penetrated deeper into the tissue, the more intense homogenous signals from the RPE and choroid overshadowed these NFL features. In addition, because visible spectrum has shorter wavelengths, it has arguably stronger backscattering from NFL, which also increases its relative intensity in the fundus image. To verify, we measured relative intensity ratio between the NFL and its immediate surrounding tissues 

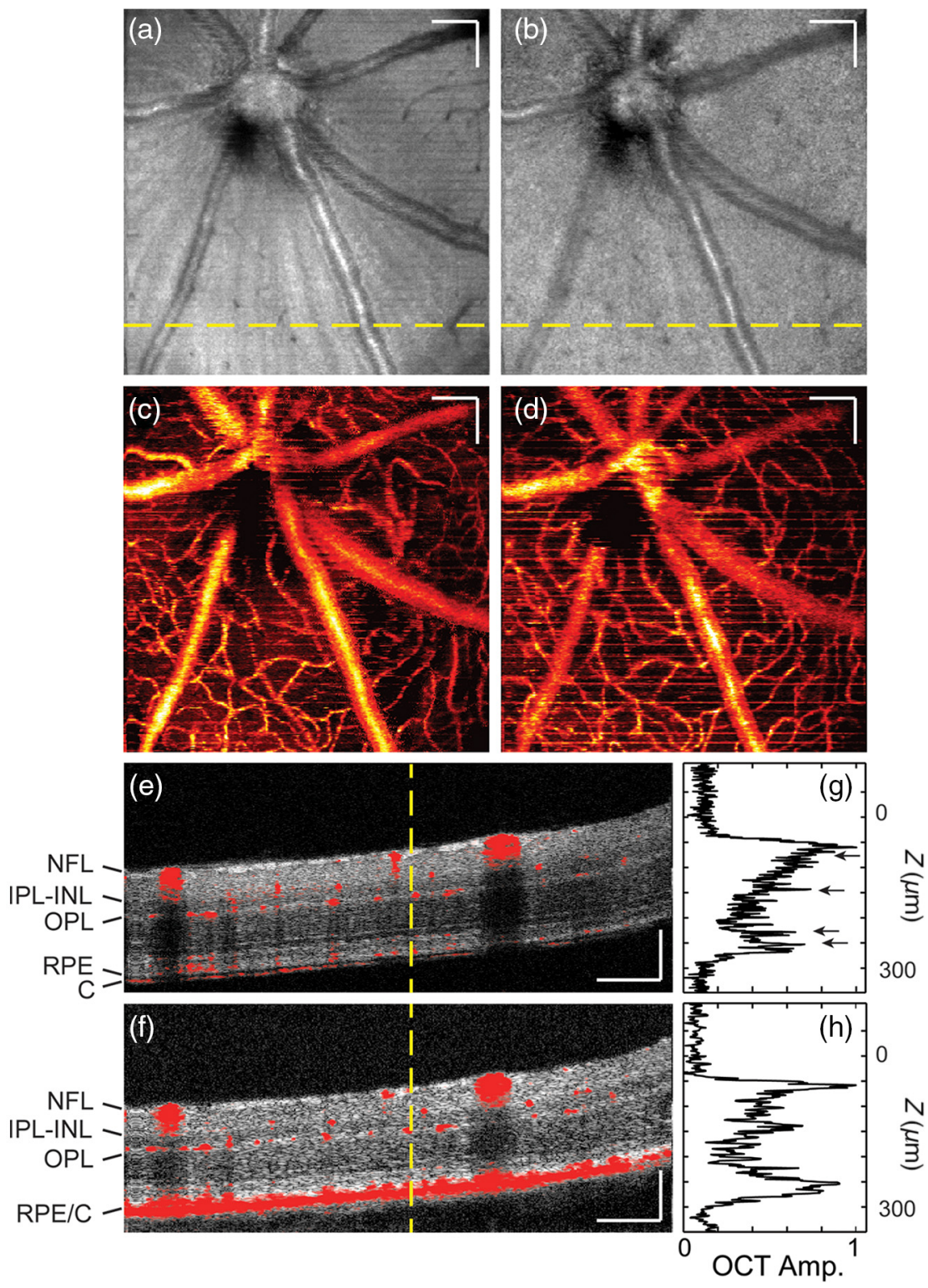

Fig. 3 Comparison of Vis- and NIR-OCT structural and angiographic images. (a) and (b) En face view of OCT structure image for Vis- and NIR-OCT, respectively. (c) and (d) En face view of OCT angiography for Vis- and NIR-OCT, respectively. (e) and (f) Merged B scan showing both structure and blood vessel, taken from the location indicated by the yellow dashed line in (a) and (b), respectively. C, choroid; IPL-INL, inner plexiform and inner nuclear layer; NFL, neural fiber layer; OPL, outer plexiform layer; RPE, retinal pigment epithelium. (g) and (h) A-line profiles taken from yellow dashed line in (e) and (f), respectively. Scale bar: $100 \mu \mathrm{m}$.

from the displayed B-scans, which averaged to 3.2:1 and 2.0:1 for visible and NIR images, respectively.

Our Vis-OCT demonstrated superior axial resolution over NIR-OCT, as seen in Fig. 3(e). We can distinguish RPE and choroidal layers in the Vis-OCT B-scan image. In contrast, these layers merge in Fig. 3(f) as one single highly reflective interface. We extracted A-line profiles [Figs. 3(g) and 3(h)] from the corresponding locations highlighted by the yellow dashed lines. We identified the profile peaks by black arrows in Fig. 3(g). These peaks, corresponding to the retinal layer interfaces, are narrower than those similar features presented in Fig. 3(h).

In addition, Figs. 3(e) and 3(f) demonstrated drastically different quality of speckle patterns. While the speckles in the
Vis-OCT B-scan are more densely packed and scattered, they appeared to be larger and clumped in the NIR-OCT image. The difference can be explained by the longer coherence length of the NIR illumination light and the fact that the NIR subsystem has lower imaging resolution than the visible subsystem. ${ }^{22}$

One weakness of Vis-OCT is its limited penetration depth. Due to stronger tissue absorption and scattering in the visible spectral range, visible light energy dissipates faster than NIR. This was evident in the A-line profiles as Vis-OCT signal demonstrated stronger attenuation across the retina thickness. Though both subsystems have adequate penetration depth to image the entire neural retina in our current application, NIR would be the preferred choice if the target of interest lies beneath RPE. 


\subsection{Comparison of Oximetry Capability Between Vis- and NIR-OCT}

We compared the abilities to extract retinal blood $\mathrm{sO}_{2}$ using our visible-light and NIR dual-band OCT. Figures 4(a) and 4(b) show the wavelength-dependent OCT intensity taken from all identified major arteries (four counts) and veins (four counts) from Vis- and NIR-OCT raster scanning data sets, respectively. The arterial and venous OCT intensity spectra show two clear crossings in the visible range. This guaranteed the convergence of the least-squares fitting algorithm. As the accuracy and reliability of Vis-OCT oximetry have recently been demonstrated in both ex vivo blood sample and in vivo rodents, ${ }^{13}$ here we only present the averaged $\mathrm{sO}_{2}$ (mean \pm S.D.), giving $\mathrm{sO}_{2}=98 \pm$ $3 \%$ for arterial blood and $\mathrm{sO}_{2}=85 \pm 3 \%$ for venous blood, respectively.

In contrast, the two corresponding spectra from NIR-OCT data set show little difference in their spectral distributions [Fig. 4(b)]. Even though theoretically there exists one isosbestic point at around $822 \mathrm{~nm}$ in the oxygenated/deoxygenated whole blood absorption spectrum, ${ }^{23}$ such feature was not identifiable in NIR-OCT measurements. One important confounding factor is that blood scattering coefficients are more than 100 times stronger than absorption coefficients in the NIR wavelength range, but they have less usable differentiating contrast. ${ }^{23}$ As a single scaling factor can almost convert one spectrum to another, a mathematical interpretation that correlates such spectral changes with $\mathrm{sO}_{2}$ is much more difficult. In this case, our least-squares regression failed to converge and we did not obtain reliable $\mathrm{sO}_{2}$ estimations, which agreed with our numerical simulation studies. ${ }^{8}$

\subsection{Comparison of Flowmetry Capability Between Vis- and NIR-OCT}

Finally, we demonstrated that both Vis-OCT and NIR-OCT subsystems can accurately measure absolute blood flow in the retinal circulation. Figures 5(a) and 5(b) show the B-scan anatomical images overlaid with $\Delta \varphi$ along the trajectory of the smaller ring scan for Vis-OCT and NIR-OCT, respectively. The directions of blood flow show an alternating pattern as expected, reflecting the anatomical arrangement of arteries and veins within the retinal circulation. In vessel numbers $6,8,9$ and 10 , phase wrapping can be observed, possibly due to higher blood flow rate or smaller Doppler angle. The phenomenon is more pronounced in the Vis-OCT image, Fig 5(a). This can be explained by the fact that Vis-OCT used the shorter range of the light spectrum, rendering it more susceptible to the $2-\pi$ ambiguity.
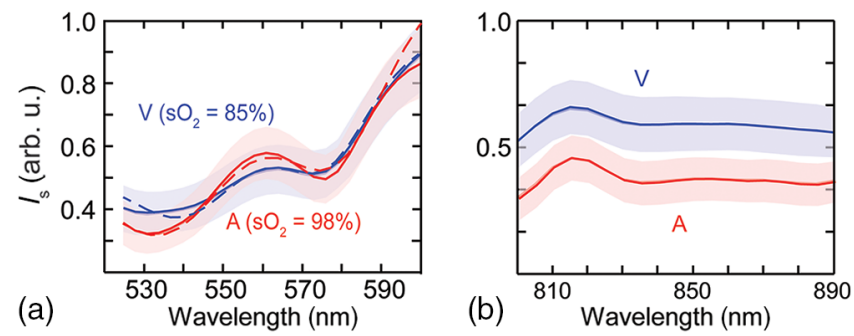

Fig. 4 Extracted averaged OCT intensity spectra from within all identified arteries and veins. (a) Intensity spectra from the Vis-OCT subsystem. Dashed lines depict fitted spectra. (b) Intensity spectra from the NIR-OCT subsystem. Shaded area $=$ S.E.M.
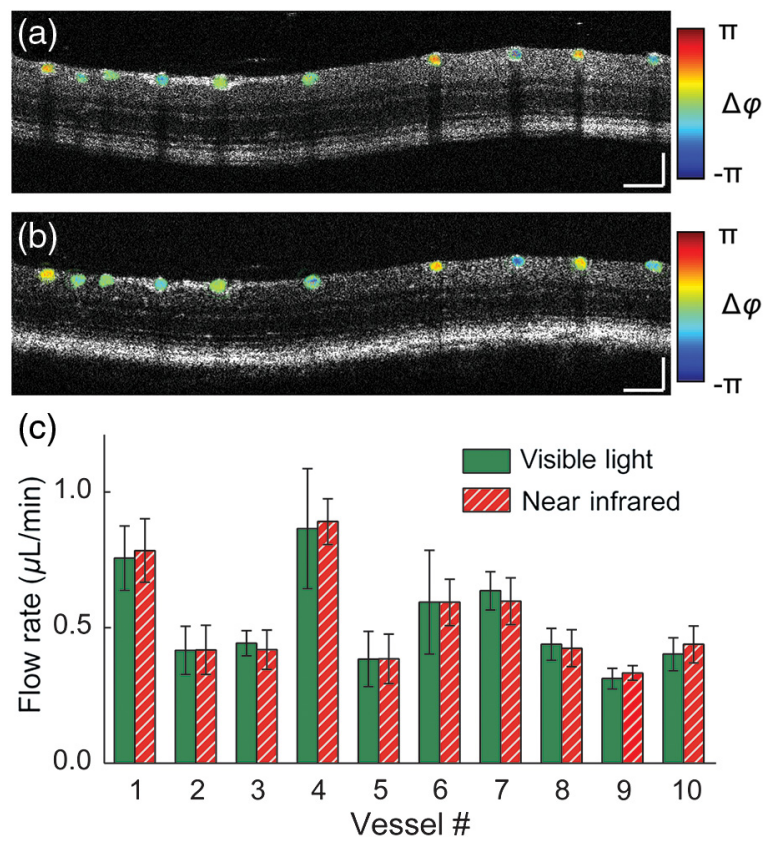

Fig. 5 Doppler OCT flow measurement. (a) and (b) B-scan structure image overlaid with phase variation, obtained using (a) Vis-OCT and (b) NIR-OCT, respectively. (c) Measured absolute blood flow in each vessel shown, numbered from left to right. Scale bar: $100 \mu \mathrm{m}$.

We calculated the absolute flow rate within each identified major blood vessel and compared them in Fig. 5(c). The presented results were averaged values (mean \pm S.D.) obtained from eight consecutive pairs of dual-ring scans. The total flow rate was $2.71 \pm 0.27 \mu \mathrm{L} / \min$ for arteries and $2.53 \pm 0.27 \mu \mathrm{L} /$ min for veins as measured by Vis-OCT. Our NIR-OCT provided similar values of $2.76 \pm 0.17$ and $2.52 \pm 0.17 \mu \mathrm{L} / \min$ for arteries and veins, respectively. We performed two-sample Student's $t$-test using respective flow values. No statistically significant difference was found between total blood flow of arteries and veins ( $p=0.56$ for Vis-OCT and $p=0.32$ for NIROCT) or between Vis- and NIR-OCT measurements ( $p=0.89$ for arterial flow and $p=0.96$ for venous flow). The consistency of these values indicated that our measurements were reliable and that Vis- and NIR-OCT had comparable performances in blood flow measurements.

\section{Conclusions and Future Perspectives}

Understanding how Vis-OCT imaging capability differs from that of the more established NIR-OCT was one fundamental step toward its potential clinical applications. In the presented study, we constructed a visible-light and NIR dual-band OCT system using a single supercontinuum laser. Sharing the same light source and the majority of imaging optics, our setup eliminated many confounding factors associated when switching between two imaging systems. Thus, it offered a fair and straightforward comparison between Vis- and NIR-OCT.

We demonstrated that both Vis- and NIR-OCT images showed reasonably similar anatomical and angiographic structures when imaging the mouse retina. However, only Vis-OCT was able to differentiate the RPE-choroid complex due to its higher axial resolution. Such a feature has the potential to facilitate RPE pathognomonic evaluation. ${ }^{24}$ Other discrepancies also 
existed, which were largely due to the distinctive absorption and scattering properties within the two bands. First, visible light attenuated and dissipated quickly along the depth, so it could barely image structures beneath the RPE layer. On the contrary, NIR-OCT offered more uniform illumination of the entire retina thickness, enabling greater imaging depth. Second, Vis-OCT images showed higher contrast within NFL. It might give Vis-OCT a unique advantage when changes in NFL thickness and reflectivity are of clinical significance, such as in the diagnosis of glaucoma. ${ }^{25}$

In terms of OCT flowmetry, both systems offered a reliable measurement of absolute blood flow within the retina circulation. However, due to the shorter wavelength used, Vis-OCT is more vulnerable to the $2-\pi$ phase-wrapping and needs more careful treatment. Based on our observation, we recommend that NIR-OCT is the preferred choice for flow measurement applications.

Finally, we verified that Vis-OCT can resolve the spectral contrast between oxygenated and deoxygenated blood, resulting in robust $\mathrm{sO}_{2}$ estimation in the retina circulation. It was the only aspect tested where NIR-OCT failed to offer comparable results. Combined with blood flow values, Vis-OCT $\mathrm{sO}_{2}$ measurement can provide noninvasive assessment on the oxygen metabolic rate of the retina. As these retinal neurons are highly metabolically active and almost entirely rely on the moment-to-moment delivery of oxygen, abnormalities in oxygen supply will surely lead to dysfunctions. ${ }^{26}$ Detecting these abnormalities in retinal oxygen supply could shed light on retina physiological and pathological investigation, as well as provide a potential biomarker for early-stage disease detection and late-stage disease management. 27,28

We would like to point out that our NIR-OCT subsystem had a higher noise level than commercial systems or other laboratorial prototypes. This is evident as our signal-to-noise ratio (SNR) was only $98.2 \mathrm{~dB}$ instead of a detector shot-noise limited SNR $(\sim 120 \mathrm{~dB}) .{ }^{29}$ As the major contribution factor is associated with the elevated relative intensity noise inherited from the supercontinuum laser, change to a less noisy source such as a super luminous diode will improve the SNR. However, similar sources are not currently available in the visible range and any improvements made are solely associated with the source property rather than the optical wavelength range used. In order to make a fair comparison between the performances of the Visand NIR-OCT systems, we decided to use the same light source for both systems.

Aside from the performance comparison, there are still other concerns regarding future clinical applications of Vis-OCT. One key consideration is the potential discomfort in patients caused by visible-light illumination. However, such discomfort may be eased by limiting the light intensity and continuously raster scanning the probing beam across the retina, as reported in a recent publication on a clinical Vis-OCT system for human subjects. ${ }^{30}$ We can also potentially minimize light exposure by decreasing the acquisition time, which includes the use of more sensitive detectors as they become available and designing more efficient scanning strategy.

In summary, both Vis- and NIR-OCT are versatile imaging modalities capable of providing invaluable information in ophthalmology applications. They complement, rather than compete, each other to provide a wide array of assessment on the anatomy and functionality of the retina. While the wavelength bands of choice will still be based on the specific requirements of the application, a dual-band OCT system similar to the one developed can provide a compact solution and offer a broader range of information than either one alone.

\section{Acknowledgments}

This work is supported in part by the following agencies: National Institutes of Health (1R01EY019951, 1R24EY022883, 1DP3DK108248) and National Science Foundation (CBET1055379, DBI-1353952). H. F. Zhang has financial interests in Opticent Health, which, however, did not support this work.

\section{References}

1. A. F. Fercher, "Optical coherence tomography-development, principles, applications," Zeitschrift für Medizinische Physik 20(4), 251276 (2010).

2. J. M. Schmitt, "Optical coherence tomography (OCT): a review," IEEE J. Sel. Topics Quantum Electron. 5(4), 1205-1215 (1999).

3. A. F. Fercher et al., "Optical coherence tomography-principles and applications," Rep. Prog. Phys. 66(2), 239-303 (2003).

4. A. Harris et al., "A review of methods for human retinal oximetry," Ophthalmic Surg. Lasers Imaging Retina 34(2), 152-164 (2003).

5. L. Kagemann et al., "Spectral oximetry assessed with high-speed ultrahigh-resolution optical coherence tomography," J. Biomed. Opt. 12(4), 041212 (2007).

6. D. J. Faber et al., "Toward assessment of blood oxygen saturation by spectroscopic optical coherence tomography," Opt. Lett. 30(9), 10151017 (2005).

7. D. J. Faber and T. G. van Leeuwen, "Are quantitative attenuation measurements of blood by optical coherence tomography feasible?," Opt. Lett. 34(9), 1435-1437 (2009).

8. S. Chen et al., "Monte Carlo investigation of optical coherence tomography retinal oximetry," IEEE Trans. Biomed. Eng. 62(9), 2308-2315 (2015).

9. S. Prahl, "Optical absorption of hemoglobin," 1999, http://omlc.ogi.edu/ spectra/hemoglobin/index.html (September 2015).

10. N. Bosschaart et al., "In vivo low-coherence spectroscopic measurements of local hemoglobin absorption spectra in human skin," $J$. Biomed. Opt. 16(10), 100504 (2011).

11. F. E. Robles et al., "Molecular imaging true-colour spectroscopic optical coherence tomography," Nat. Photonics 5(12), 744-747 (2011).

12. J. Yi et al., "Visible-light optical coherence tomography for retinal oximetry," Opt. Lett. 38(11), 1796-1798 (2013).

13. J. Yi et al., "Visible light optical coherence tomography measures retinal oxygen metabolic response to systemic oxygenation," Light Sci. Appl. 4(9), e334 (2015).

14. S. P. Chong et al., "Quantitative microvascular hemoglobin mapping using visible light spectroscopic optical coherence tomography," Biomed. Opt. Express 6(4), 1429-1450 (2015).

15. S. P. Chong et al., "Cerebral metabolic rate of oxygen $\left(\mathrm{CMRO}_{2}\right)$ assessed by combined Doppler and spectroscopic OCT," Biomed. Opt. Express 6(10), 3941-3951 (2015).

16. B. Povazay et al., "Visible light optical coherence tomography," Proc. SPIE 4619, 90-94 (2002).

17. Y. Wang et al., "Retinal blood flow measurement by circumpapillary Fourier domain Doppler optical coherence tomography," J. Biomed. Opt. 13(6), 064003 (2008).

18. J. Lee et al., "Motion correction for phase-resolved dynamic optical coherence tomography imaging of rodent cerebral cortex," Opt. Express 19(22), 21258-21270 (2011).

19. S. Chen, J. Yi, and H. F. Zhang, "Measuring oxygen saturation in retinal and choroidal circulations in rats using visible light optical coherence tomography angiography," Biomed. Opt. Express 6(8), 2840-2853 (2015).

20. D. C. Ghiglia and M. D. Pritt, Two-Dimensional Phase Unwrapping: Theory, Algorithms, and Software, Wiley, New York (1998).

21. H. Li, W. Liu, and H. F. Zhang, "Investigating the influence of chromatic aberration and optical illumination bandwidth on fundus imaging in rats," J. Biomed. Opt. 20(10), 106010 (2015). 
22. J. M. Schmitt, S. H. Xiang, and K. M. Yung, "Speckle in optical coherence tomography," J. Biomed. Opt. 4(1), 95-105 (1999).

23. N. Bosschaart et al., "A literature review and novel theoretical approach on the optical properties of whole blood," Lasers Med. Sci. 29(2), 453-479 (2014).

24. X. Shu, W. Liu, and H. F. Zhang, "Monte Carlo investigation on quantifying the retinal pigment epithelium melanin concentration by photoacoustic ophthalmoscopy," J. Biomed. Opt. 20(10), 106005 (2015).

25. S. Liu et al., "Retinal nerve fiber layer reflectance for early glaucoma diagnosis," J. Glaucoma 23(1), e45-e52 (2014).

26. C. Caprara and C. Grimm, "From oxygen to erythropoietin: relevance of hypoxia for retinal development, health and disease," Prog. Retin. Eye Res. 31(1), 89-119 (2012).
27. H. P. Hammes, "Pericytes and the pathogenesis of diabetic retinopathy," Horm. Metab. Res. 37(S 1), 39-43 (2005).

28. M. Mozaffarieh, M. C. Grieshaber, and J. Flammer, "Oxygen and blood flow: players in the pathogenesis of glaucoma," Mol. Vis. 14, 224-233 (2008).

29. M. A. Choma et al., "Sensitivity advantage of swept source and Fourier domain optical coherence tomography," Opt. Express 11(18), 2183-2189 (2003).

30. J. Yi et al., "Human retinal imaging using visible-light optical coherence tomography guided by scanning laser ophthalmoscopy," Biomed. Opt. Express 6(10), 3701-3713 (2015).

Biographies for the authors are not available. 